\title{
Side Effects and Biochemical Changes in Rat Liver on Drug Treatment
}

\author{
Rajesh K. Bhaskar \\ School of Biosciences., Mahatma Gandhi University, Priyadarsini Hills P.O., Kottayam, Kerala , India
}

\begin{abstract}
Antibiotics are the therapeutic agents most often associated with hepatotoxicity mainly due to the widespread prescription of these drugs. Causality assessment of suspected drug-induced liver injury (DILI) related to antibiotics can be difficult, particularly because some cases occur long after the drug has been stopped. Among the penicillins, amoxicillin clavulanate is the most associated with hepatotoxicity and is the most frequent cause of DILI-related hospitalisations. Flucloxacillin ranks as the second highest cause of DILI in many countries. The severity of antibiotic-induced DILI varies widely, with the hepatitis-like (hepatocellular) damage tending to be more severe that than cholestatic/mixed type. The pattern is strongly influenced by age. Recently telithromycin (a new generation macrolide) has been linked with DILI, with a typical pattern, which includes abrupt commencement of fever, abdominal pain, jaundice and, in some cases, ascites. Antibiotic-induced DILI appears to be idiosyncratic. Genetic-association studies have recently identified genotypes related to flucloxacillin and possibly to amoxicillin-clavulanate hepatotoxicity.
\end{abstract}

Keywords: Drug-induced liver injury (DILI), antibiotics, fulminant liver failure,hepatotoxixicty, causality assessment, amoxicillinclavulanate, genetic factors.

\section{Introduction}

The liver is the main site of metabolism for drugs and other exogenous compounds. As most drugs are taken orally the liver is the portal to the tissues for such compounds following absorption from the gastrointestinal tract. The liver is, therefore, a vulnerable organ, being exposed to both the parent drug carried from the G.I. tract via the portal vein and to any metabolites produced which then enter the systemic circulation via the hepatic vein. However, despite this vulnerability, the liver is not the major target for adverse drug reactions, only about $9.5 \%$ of these involve the liver (Davis and Williams,1977). Although drug-induced hepatic damage may not be particularly common in general patient populations the case fatality rate is often high, and the severity of drug-induced hepatic injury is such that drugs are a major cause of hepatic failure( Zimmerson,1978). For example, the case fatality rate from halothane-induced hepatic failure may be as high as $50 \%$.Chemical agents such as those used in laboratories and industries, natural chemicals (e.g. microcystins) and herbal remedies can induce hepatotoxicity.

Chemicals that cause liver injury are called hepatotoxins. More than 900 drugs have been implicated in causing liver injury. chemicals often cause subclinical injury to liver which manifests only as abnormal liver enzyme tests. Drug induced liver injury is responsible for $5 \%$ of all hospital admissions and $50 \%$ of all acute liver failures ( Ostapowicz et al,2002).Drug related hepatotoxicity is an important cause of morbidity and mortality and indeed is the most common reason for withdrawing new drugs.Drugs and other exogenous compounds may affect the liver in various ways. Hepatotoxicity is one of the most important adverse drug reactions associated with anti-tuberculosis drugs that may limit their use. Aspirin and the salicylates have recently been recognized as potentially hepatotoxic (Prescott,1980). Phenylbutazone can cause acute liver injury without involvement of other systems with therapeutic doses and following over dosage. In this review various types of drug induced hepatotoxicity, mechanisms of drug induced hepatotoxicity and various class of drugs used in different clinical therapies causing hepatotoxicity.

\section{Types of Drug Induced Hepatotoxicity}

Drug-induced liver injury covers a variety of types, and includes most of the clinical and pathological expressions of liver damage ( Zimmerman,1978).

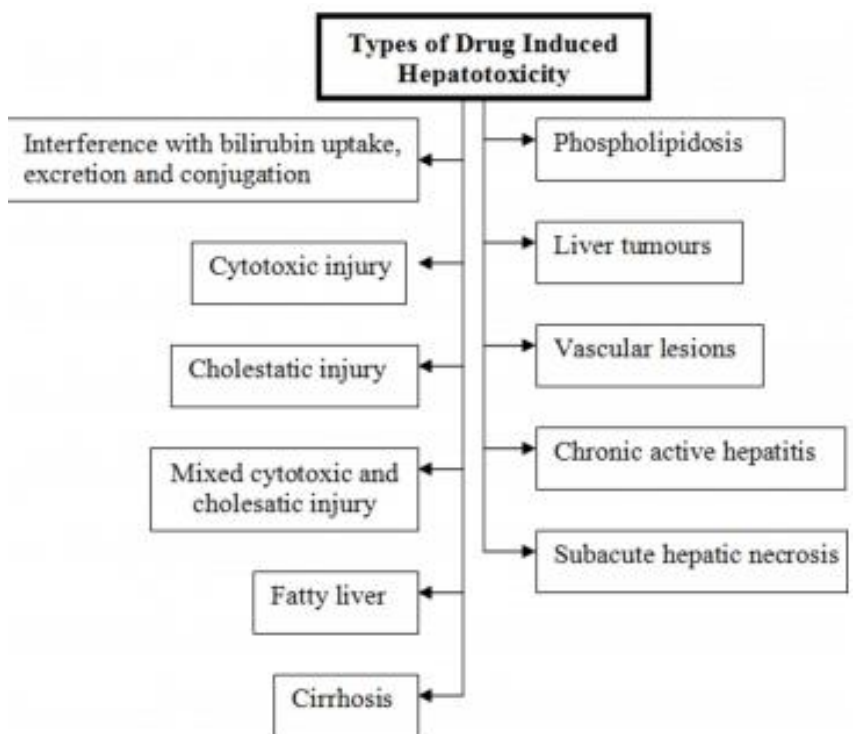

Figure 1: Types of drug induced hepatotoxicity

1) Interference with bilirubin uptake, excretion and conjugation:This may be viewed as a variant of cholestatic toxicity. For example; Rifampicin may interfere with bilirubin transport giving rise to hyperbilirubinaemia ( Capelle et al,1972).

2) Cytotoxic injury:damage to the parenchyma and is a much more serious effect than the above (Timbrell,1983).

3) Cholestatic injury: This type involves arrested bile flow and jaundice and may appear similar to biliary 


\section{International Journal of Science and Research (IJSR) \\ ISSN (Online): 2319-7064}

Index Copernicus Value (2013): 6.14 | Impact Factor (2014): 5.611

obstruction. It is less serious than cytotoxic injury, with a lower fatality rate.

4) Mixed cytotoxic and cholesatic injury: Cytotoxic hepatic damage may sometimes be combined with cholestasis, for instance occasionally seen after paminosalicylic acid therapy ( Zimmerman,1974 ).

5) Fatty liver: Fatty liver (steatosis) may be considered as a type of cytotoxic injury, but it may also be a form of chronic hepatic damage.

6) Cirrhosis: Macronodular cirrhosis may directly follow acute hepatic damage and primary biliary cirrhosis may result from cholestatic jaundice.

7) Phospholipidosis: This may result from the use of drugs such as Coralgil,(4, 4'-diethylamino ethoxyhexestrol dihydrochloride), and is characterised by hepatocytes full of lipid(Lulluman et al,1975).

8) Liver tumours: Neoplastic lesions may result from drug use. Benign, liver cell adenomas have been associatedwith the use of contraceptive steroids (Neuberger, 1981) but liver cell carcinoma associated with these drugs is more uncertain.
9) Vascular lesions: Hepatic vein occlusion, such as that due to the thrombogenic effect of contraceptive steroids, may result in hepatic damage.

10) Chronic active hepatitis: progressive necroinflammatory liver disease which may have many causes including drugs.

11) Subacute hepatic necrosis: syndrome consists of progressive, overt serious liver disease with jaundice and eventual cirrhosis.

\section{Mechanisms \\ of Drug \\ Induced Hepatotoxicity}

Certain drugs will produce predictable liver damage in the majority of cases, such as after overdoses, for instance. Others however will cause liver injury only rarely and unpredictably. There is thus a whole spectrum varying between these extremes. In some cases the mechanism may involve the parent compound; in others a metabolite may be responsible.

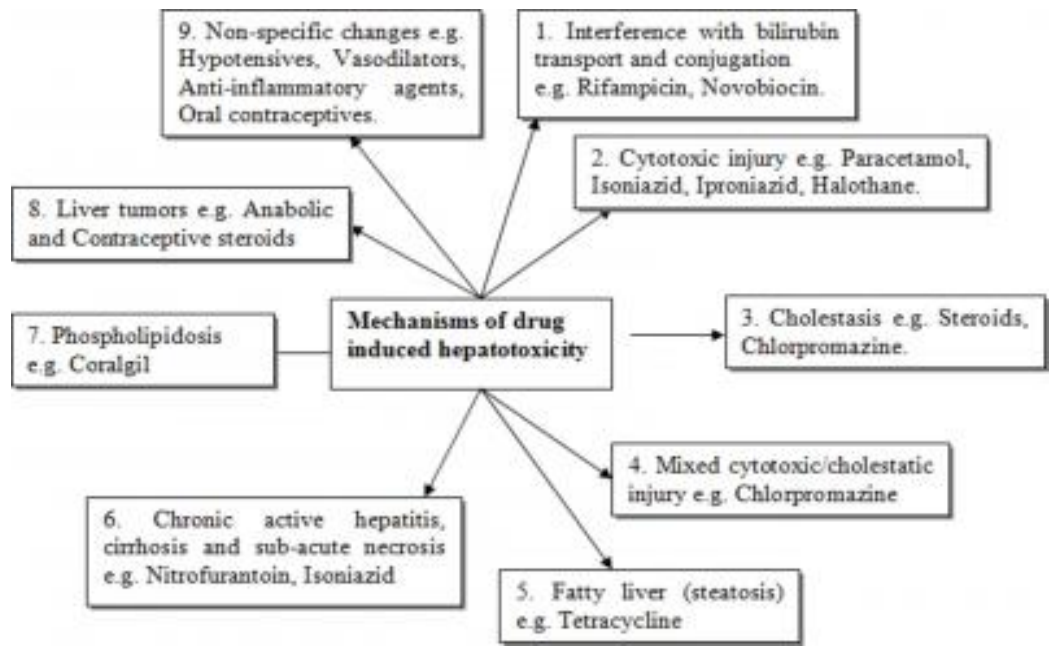

Figure 2: Mechanisms of drug induced hepatotoxicity

1. Interference with bilirubin transport and conjugation: A number of drugs interfere with bilirubin transport and lead to elevated plasma bilirubin or hyperbilirubinaemia. Novobiocin inhibits UDP glucuronosyl transferase and may lead to elevated plasma levels of unconjugated bilirubin especially in neonates (Breen et al, 1972). Rifampicin, the antibiotic used in the treatment of tuberculosis inhibits both uptake and excretion of bilirubin in a dose related manner, giving rise to elevated plasma levels of both conjugated and unconjugated bilirubin. This is due to blockade of uptake at the plasma membrane of the hepatocyte ( Mitchel et al,1975 ).

2. Cytotoxic injury: Direct, overt damage to hepatic parenchyma may be caused by a number of drugs. However, it may have a variety of underlying mechanisms. Paracetamol causes predictable centrilobular hepatic necrosis in experimental animals as well as man after overdoses (Bahari et al, 1981 and Lullman and Rauch, 1977).It is well characterized and the mechanism partially understood. The liver damage is predictable and is due to direct cytotoxicity of a metabolite as indicated by extensive studies on both experimental animals (Goldfarb, 1976) and man (Rao, 1973). Paracetamol is metabolised by three pathways, (Figure 3), two of which are conjugation reactions, and remove the major portion of the drug rapidly. After overdoses however, the amount of reactive metabolite is sufficient to deplete the available hepatic glutathione. The reactive metabolite then reacts covalently with cellular macromolecules. The mechanism by which this binding causes cellular damage is unknown. Isoniazid and iproniazid which are substituted hydrazine drugs may produce a hepatocellular, cytotoxic type of damage.

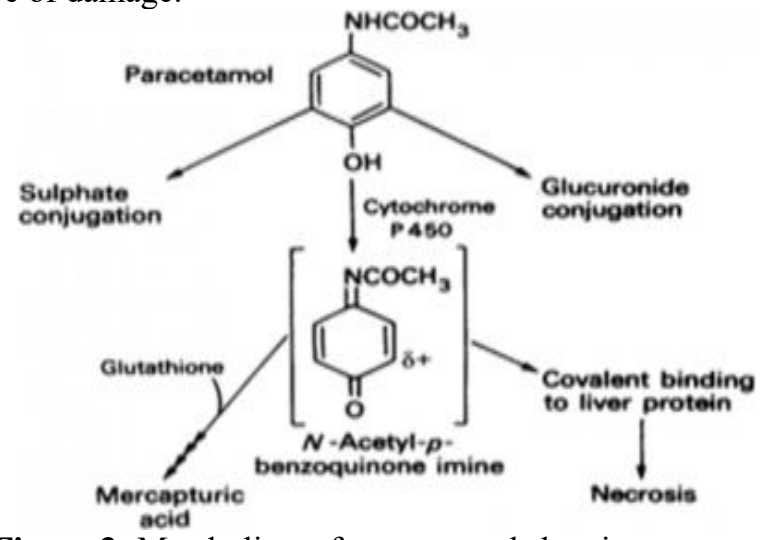

Figure 3: Metabolism of paracetamol showing proposed metabolic activation and its involvement in the toxicity

\section{Volume 5 Issue 2, February 2016}




\section{International Journal of Science and Research (IJSR) \\ ISSN (Online): 2319-7064}

Index Copernicus Value (2013): 6.14 | Impact Factor (2014): 5.611

3. Cholestasis: This type of reaction is the result of inability of the hepatocyte to secrete bile due to impaired bile salt secretion ( McClain et al,1999). Drug induced cholestatic reactions are classified as -

\section{1] Steroid-induced cholestasis and 2] Sensitivity type of cholestasis.}

\section{a) Steroid-induced cholestasis}

E.g. C-17 substituted testosterones including orally active anabolic and androgenic agents and oral contraceptives. The jaundice induced by steroids is usually mild and reversible when the drug is stopped ( Schaller,1978). The reaction is dose related and develops after an initial period of medication. Bromsulphathelein (BSP) excretion is usually impaired. This reaction is predicted by -

1) Structure of the compound - C-17 substituted testosterone.

2) BSP excretion curves in man and rat.

3) Electron microscopic examination of the rat liver following the administration of the drug would reveal an appreciable number of canaliculi changes such as dilation and loss of microvilli (Cheuk - ming,2002).

\section{b) Sensitivity type of cholestasis}

This type of reaction is usually associated with the phenothiazines like chlorpromazine, trifluoperazine, promazine, pecazine, etc. It is not dose-related and develops after an initial period of sensitization of 1-4 weeks or previous exposure. The common symptoms noticed are rashes, fever, eosinophilia and blood dyscrasias. The jaundice simulates surgical obstructive type, lasts for 1-4 weeks and recovery is complete.

\section{Mixed cytotoxic/cholestatic injury}

This type of liver injury covers damage with varying proportions of cytotoxic and cholestatic involvement. For example chlorpromazine may cause mixed hepatocanalicular jaundice with parenchymal injury as well as cholestasis. Conversely, p-aminosalicylic acid may cause mixed hepatocellular liver injury.

\section{Fatty liver (steatosis)}

Tetracycline is an antibiotic which may occasionally cause fatty liver after large ( $1.5 \mathrm{~g}$ /day) intravenous doses. This toxic effect is very rare after oral doses and occurs more commonly in females than males. This toxic effect of tetracycline is direct, predictable and dose dependent, and can be reproduced in experimental animals. The major effect seems to be inhibition of transport of lipid out of the hepatocyte, which can be detected within 30 min of dosing in experimental animals. This effect may well be due to the inhibition of protein synthesis caused by tetracycline which will inhibit the production of the apolipoprotein complex involved in transport of the very low density lipoprotein (VLDL) out of the hepatocyte (Sharma et al,2002). Alternative or additional mechanisms may involve decreased fatty acid oxidation, increased triglyceride uptake or increased fatty acid uptake. (Figure 4).
6. Chronic active hepatitis, cirrhosis and sub-acute necrosis

Chronic active hepatitis, sometimes associated with cirrhosis is associated with the use of a number of drugs such as oxyphenisatin a-methyldopa, nitrofurantoin and isoniazid. Long term administration of the antitubercular drug isoniazid leads to hepatic dysfunction in a significant proportion of recipients (10-20\%). This is usually mild and subclinical and subsides despite continued therapy. The mechanism of isoniazid induced hepatic damage involves production of a toxic metabolite. Mitchell et al (1975) suggested that rapid acetylation might be a predisposing factor as it was reasoned that this would produce more of the metabolite acetylisoniazid and hence more acetylhydrazine.

\section{Phospholipidosis}

This syndrome may be caused by a number of different drugs, and various organs may be affected. Hepatic phospholipidosis has been caused by the drug Coralgil, a coronary dilator. The features of this form of hepatic damage are an accumulation of phospholipids in hepatocytes, bile duct proliferation and inflammation in the portal area. The mechanism is thought to involve the formation of complexes between lipid micelles or liposomes, and the drug in question.

\section{Liver tumors}

Anabolic steroids have been implicated as responsible for primary hepatocellular carcinomas and adenomas. Similarly use of contraceptive steroids has been connected with liver tumours, particularly the oestrogenic components. (Bruchfeld, 2000).

\section{Non-specific changes}

Certain hypotensives, vasodilators, anti-inflammatory agents and oral contraceptives cause a transient increase in transaminase levels which revert to normal level after the drug is stopped. These reactions cannot be predicted (Compos-Franco, 2004).

\section{Drugs Causing Hepatotoxicity}

1. Analgesics: Acetaminophen (paracetamol) is among the most commonly used analgesics. It effectively reduces fever and mild to moderate pain, and is regarded, in general, as a very safe drug. Hepatic injury with acetaminophen is not only associated with overdose or use of high doses; rather, it can be encountered with chronic use at lower doses $(<4 \mathrm{~g} /$ day), particularly in the presence of other predisposing factors, such as chronic alcohol consumption ( Barness, 1993). Damage to the liver following acetaminophen ingestion is not due to the drug itself, but due to a toxic metabolite that is generated through the cytochrome $\mathrm{P}_{50}$ group of enzymes in the liver. This metabolite is usually rendered harmless through an interaction with the endogenous antioxidant, glutathione. However, when there is overproduction of the acetaminophen metabolite, glutathione stores in the liver become depleted, and the metabolite begins to accumulate and cause tissue injury. Hepatic injury can be limited through administration of $\mathrm{N}$-acetylcysteine, which replenishes liver levels of glutathione. Aspirin has been involved in the great majority of cases. About $50 \%$ of 


\section{International Journal of Science and Research (IJSR) \\ ISSN (Online): 2319-7064}

Index Copernicus Value (2013): 6.14 | Impact Factor (2014): 5.611

patients with juvenile rheumatoid arthritis have evidence of some degree of liver injury as shown by elevation of plasma aminotransferases during conventional high-dosage aspirin therapy ( Kimmon and Samuel,2002 ). Other drug in this category includes gabapentin which shows hepatotoxicity as one of its side effects ( Jainne et al,1998).

2. Anti-tuberculosis drugs:Hepatotoxicity is one of the most important adverse drug reactions associated with antituberculosis drugs that may limit their use. Previous studies showed transient elevations of serum hepatocellular enzymes (e.g. alanine aminotransferase and aspartate aminotransferase) in approximately $10 \%$ of patients who received a standard combination chemotherapy including isoniazid and rifampicin, of these 1-2\% patients withdrew from the treatment because of severe hepatotoxicity that ultimately led to fulminant hepatitis. Although the occurrence of drug induced hepatotoxicity is difficult to predict, it has been observed that certain patients are at higher risk during the course of anti-TB chemotherapy ( Stolk et al,2006 and Chitturi \& George,2002).

3. Anti-hyperlipidemics: The anti-hyperlipidemic drug with the highest potential for hepatic injury is the sustainedrelease formulation of niacin. $\mathrm{HMG} \mathrm{CoA}$ reductase inhibitors, otherwise known as statins, very rarely cause clinically significant liver injury, although asymptomatic elevation in aminotransferases is common. The notion that ezetimibe may have less risk of hepatotoxicity has recently been challenged and it may not be a "safe alternative" to statins in patients with pre-existing liver disease(Hodis, 1990).

1) HMG CoA reductase inhibitors (Statins):Initial studies of statins performed on animals revealed that very high doses of statins may cause hepatotoxicity, but typical therapeutic doses of the drug were not associated with significant liver injury (Nickerson \& Ruedy,1975) High doses of lovastatin caused significant hepatocellular necrosis in rabbits.

a) Atorvastatin:Atorvastatin-related hepatotoxicity has been associated with a mixed pattern of liver injury typically occurring several months after the initiation of the medication.

b) Lovastatin: Mixed hepatic injury in hepatocellular and cholestatic patterns has been noted with the use of lovastatin.

c) Simvastatin:Simvastatin hepatotoxicity is hypothesized to occur due to drug-drug interactions.

d) Pravastatin:Pravastatin has been reported to cause acute intrahepatic cholestasis. In this case, liver toxicity occurred within 2 months after initiating the drug and it resolved within 2 months after its discontinuation.

2) Niacin: Unsupervised use of the sustained-released formulation of niacin often leads to its dose-related toxicity and should be discouraged (Tredge \&Davis, 1991.

3) Ezetimibe: Recent studies have noted that ezetimibe may rarely cause hepatotoxicity in the form of severe cholestatic hepatitis and acute autoimmune hepatitis. The mechanism of toxicity may be related to the metabolism of the drug; it is rapidly absorbed and glucuronidated, yielding an active metabolite and there is significant enterohepatic recirculation ( Kenna et al,1984).

4. Anti-hypertensive Drugs: Methyl dopa is used in the treatment of hypertension. Both minor and severe forms of liver damage have been reported in patients receiving methyl dopa. The former consists of asymptomatic, and often transient, rises of serum transaminases and according to various reports is found in two to $10 \%$ of patients receiving the drug ( Brown \&Sipes ,1977 and Kshirsagar, 2008). The liver damage, which may take the form of acute hepatitis, chronic active hepatitis or cholestasis occurs more commonly in women and there is not the same close temporal relationship between the time of onset of overt clinical hepatic injury, which in $50 \%$ of cases occurs after four weeks. This covalent binding is inhibited by a variety of agents, including glutathione, ascorbic acid and superoxide dismutase consistent with the oxidation of methyl dopa by cytochrome $\mathrm{P} 4_{50}$-generated superoxide anions to a reactive quinone.

5. Anaesthetic Agents: Halothane, the most widely used anaesthetic is now accepted as causing hepatic injury. Multiple exposures are a major factor which may predispose the patient to liver injury. Obese patients and females seem more susceptible but children and young adults less so. A series of investigations carried out in the Liver Unit identified an antibody directed against a hepatocyte surface antigen altered by a halothane metabolite. The altered antigenic determinant probably results from oxidative halothane metabolism which generates trifluroacetylated proteins. (Figure 4)

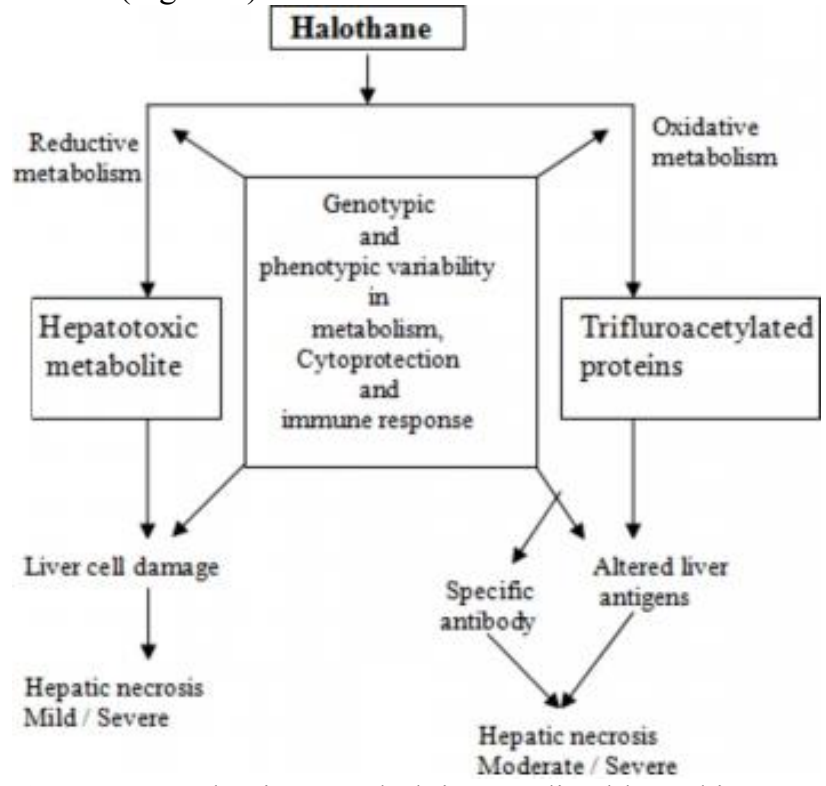

Figure 4: Mechanisms underlying predictable and immune mediated hepatotoxicity from halothane

It is likely that all individuals exposed to the drug generate altered hepatocyte membrane determinants but only a small minority mounts an immunological reaction against them . The fact that many patients with severe halothane hepatitis have circulating antibodies directed against other organs in the body strongly suggests an underlying, genetically determined defect in immune regulation. In contrast, some patients with hepatitis from the drug have no evidence of immune involvement, and liver damage in these cases is 


\section{International Journal of Science and Research (IJSR) \\ ISSN (Online): 2319-7064}

Index Copernicus Value (2013): 6.14 | Impact Factor (2014): 5.611

probably the result of overproduction of a hepatotoxic derivative produced by reductive halothane metabolism. Preferential stimulation of this pathway in experimental animals produces dose related hepatotoxicity (Kaufmann, 1995).

6. Drugs used in Anti-retroviral therapy: Giuseppe et al.(2007) reported the importance of stavudine as a possible causative agent of hepatotoxicity, even in the absence of other signs of mitochondrial toxicity. Stavudine is a drug used in anti-retroviral therapy. Highly active anti-retroviral therapy (HAART) is associated with a number of serious and potentially life-threatening adverse events, including druginduced hepatotoxicity.

\section{Experimental procedures for the study of Biochemical changes in rats}

\section{Sample Collection}

Male albino wilstar rats (150-200 g ) obtained from the Albino breeding station/Kerala Agricultural University, Mannuthy ,Thrissur ,Kerala were used in this study. Rats were divided into experimental groups, one group (normal) non trated and other groups were exposed to the drugs. They were housed in polypropylene cages $(47 \mathrm{~cm} \times 34 \mathrm{~cm} \times 20 \mathrm{~cm})$ lined with husk.It was renewed every 24 hours under a 12:12 hour light :dark cycle. The experiment was carried out in accordance with the guidelines of the Committee for the Purpose of Control and Supervision of Experiments on Animals(CPCSEA), NewDelhi ,India. The rats were killed and the liver quickly removed and examined for the liver injury (Bhaskar,2015).After 45 days of treatment, the animals were fasted for 12 hours, anaesthetized with pentobarbital sodium $(35 \mathrm{mg} / \mathrm{kg})$ and sacrificed by cervical decapitation. The blood was collected in tubes containing mixture of potassium oxalate and sodium fluoride as anticoagulant for the biochemical analysis. Liver dissected out,washed in ice-cold saline and patted dry weighed.10\% tissue homogenate prepared from liver used for various biochemical estimations.

\begin{abstract}
Analytical procedure for Preparation of tissue extract
After the end of the experimental period, the rats were starved (deprived of food) for 18 hours, overnight and sacrificed by cervical dislocation. Blood samples were collected through cardiac puncture into lithium heparin specimen bottles for biochemical analysis. The animals were subsequently sacrificed by cerevical dislocation. Liver function tests were performed on the blood samples using standard techniques. Liver were dissected out, blotted off blood, rinsed in phosphate buffered saline $(\mathrm{pH} 7.4)$ and then $10 \%$ tissue homogenate were prepared. Histopathological changes in the liver of test rats were compared to those of control. Tissues of target organs were ground in a clear mortar using phosphate buffer. It can be used for liver marker enzyme studies.
\end{abstract}

\section{Expected Resultsand Discussion for the Study}

Hepatotoxicity implies chemical-driven liver damage. The liver is a major entry organ of xenobiotics and airborne chemicals and a potential target both for the chemicals inhaled or circulating in the blood stream. The rat animals in the experimental groups were treated for drugs and chemicals (xenobiotics) may affect liver function which stimulate the activity of microsomal enzymes (eg.cyt.P450), a process known as enzyme induction. This is important in determining the degree of hepatotoxicity (Conney, 1967) in the animal study. Liver, the largest organ in the body is being evolved to maintain the body's internal milieu and also protect itself from the challenges it faces during its functioning. Liver damage is detected by the measurement of the activities of serum enzymes like AST, ALT, ALP, GGT which has been released into the blood from damaged cells, which are indicators of hepatic cell damage (Bhaskar, and Kurup,2015). It is a vital organ having diverse functions. It plays an important role not only in the metabolism, synthesis and storage but also in the detoxification of many endogenous and exogenous compounds and converting them to less toxic substances for excretion. Certain medicinal agents when taken in overdoses and sometimes even when introduced within therapeutic ranges may injure the liver. The liver plays a central role in transforming and clearing chemicals and is susceptible to the toxicity from these agents. The present review provides an overview of various drugs causing hepatotoxicity, various types of drug induced hepatotoxicity and their mechanisms.

(Bhaskar, 2015).

\section{Conclusion}

It is clear that drugs may cause a wide variety of hepatic lesions. Due to the presence of different types of cells blood has varied functions and analysis of its components helps in evaluating the abnormal conditions which create pathological conditions in a person. Therefore examination of blood and liver become significant to help in the diagnosis and treatment ofthe many diseases including cancer (Bhaskar,2012). In some cases these may be indistinguishable pathologically and biochemically from lesions due to other causes. Drug-induced hepatic damage ranges from the unpredictable and non-dose related to that occurring predictably after overdoses. Drug heptatotoxicity may involve metabolism to toxic, reactive intermediates and covalent interactions with cell constituents, interference, with membrane transport or with cellular biochemistry such as protein synthesis, or immunological mechanisms. The occurrence of hepatic damage may be modified by differences in immune responsiveness and genetic, dietary and other factors. Various clinical therapies should involve drug induced hepatotoxicity as an important parameter that will have to take into account the multi-factorial aspects of drug induced hepatic injury. Some toxicants cause direct injury to liver and others convert the chemicals into toxic substance through metabolic conversion (Bhaskar, 2015)..

\section{References}

[1] Bahri AK,Chiang CS,Timbrell JA.Acetylhydrazine hepatotoxicity.Tox applPharmac.,60:561-569,1981.

[2] Barnes PF, Barrows SA. Tuberculosis in the 1990s. Ann Intern Med. 119: 400-410.1993.

[3] Bhaskar RK.Liver as target organ of carcinogenesis by Xenobiotics.J.Biotechnol.Biomater.2(6),251.2012. 


\section{International Journal of Science and Research (IJSR) \\ ISSN (Online): 2319-7064}

Index Copernicus Value (2013): 6.14 | Impact Factor (2014): 5.611

[4] Bhaskar,RK.(2015).Hepatotoxicity induced by antibiotics in experimental animals.Jtasr.1 (4). OctoberNovember.,283-292,2015.

[5] Bhaskar,R.K. Antibiotic induced toxicity in animals. Ph.D.thesis. Mahatma Gandhi University, Kottayam,Kerala ,India.2015

[6] Bhaskar,R.K and Kurup,G.M.Biochemical Marker Studies of Acute Drug Induced Liver Injury in Rat. Jtasr; Volume 1,Issue 04, October-December; Page: 332337,2015.,2015.

[7] Breen L, Schenker S, Heimberg M. The effect of tetracycline on the hepatic secretion of triglyceride. Biochem Biophys Acta.270:74-80,1972.

[8] Brown BR,Sipes IG.Biotransformation and hepatotoxicity of halothane.Biochem Pharmacol.;26:2091-2094,1977.

[9] Bruchfeld J,Bruchfeld J,Aderaye G,Palme IB,Bjorvatn $\mathrm{B}$,Kallenius G.Sputum concentration improves diagnosis of tuberculosis in a setting with a high prevalence of HIV. Trans R Soc Trop Med Hyg.; 94:677-680,2000.

[10] Campos-Franco JA, Gonzalez-Quintela, Alende-Sixto MR. Isoniazid induced hyperacute liver failure in a young patient receiving carbamazepine. Eur J Intern Med. 2004; 15:396-397.

[11] Capelle P, Dhumeaux D, Mora M, Feldman G, Berthelot P. Effect of rifampicin on liver function in man.Gut.; 13:366-371,1972.

[12]Cheuk-ming T. Monitoring for hepatotoxicity during antituberculosis treatment. 2002.

[13] Chitturi S, George J. Hepatotoxicity of commonly used drugs: nonsteroidal anti-inflammatory drugs, antihypertensives, antidiabetic agents, anticonvulsants,lipidlowering agents, psychotropic drugs.Sem Liver Dis.;22(2):169-183,2002.

[14] Conney A.H.Pharmac. Rev.19, 317.1967.

[15] Davis M, Williams R. Hepatic Disorders. In: Davies DM, editor. Textbook of Adverse Drug Reactions, Oxford: Oxford University Press. pp 146-172,1977.

[16] Goldfarb S. Sex hormones and hepatic neoplasia. Cancer Res.; 36:2584-2588,1976.

[17] Giuseppe L, Ilaria I, Silvia C, Giuliana C, Luisa B, Salvatore C. Dideoxynucleoside HIV reverse transcriptase inhibitors and drug-related hepatotoxicity: a case report. J Med Case Rep.; 1(19). 2007.

[18] Hodis HN. Acute hepatic failure associated with the use of low-dose sustained-release niacin. Jama.264(2):181.,1990.

[19] Jaime R, Ungo, Jones D, Ashkin D, Hollender ES, Bernstein D, Albanese AP, Pitchenik AE. Antituberculosis drug induced hepatotoxicity.Am J Respir Crit Care Med.;157(6):1871-1876,1998,

[20] Kaufman PB, Wu W, Kim D, Cseke LJ..Handbook of molecular and cellular methods in biology and medicine.CRC Press, London, 1-27. 1995.

[21] Kenna JG, Neuberger J,Williams R.An enzyme-linked immunosorbent assay for detection of antibodies against halothane-altered hepatocyte antigens. J Immunol Meth.; 75:3-14,1984.

[22] Kimmoun E, Samuel D. Antituberculous drugs in patients with chronic liver disease. J Gastroenterol Hepatol. 2002; 17:408-412.

[23] Kshirsagar, A .,Y Vetal,P Ashok,P Bhosle,D Ingawale.Drug Induced Hepatotoxicity:A
Comprehensive Review. The Internet Journal of Pharmacology. Volume 7 Number 1,2008.

[24] Lullmann H, Lullmann-Rauch R. Drug induced lipidosis. In: Bundgaard H, Juul P, Kofod H, editors. Drug Design and Adverse Reactions.Copenhagen: Munksgaard.pp 29-34,1977.

[25] Lullman H, Lullman-Rauch R, Wassermann O. Drug induced phospholipidosis. CRC Crit Rev Tox.; 4:185218,1975 .

[26] McClain CJ, Price S, Barve S, Devalarja R, Shedofsky S. Acetaminophen hepatotoxicity: an update. Curr Gastroenterol Rep.; 1:42-49,1999.

[27] Mitchell JR, Thorgiersson UP, Black M, Timbrell JA, Snodgrass WR, Potter WZ, Jollow DJ, Keiser H.Increased incidence of isoniazid hepatitis in rapid acetylators:Possible relation to hydrazine metabolites. Clin Pharmac Ther. 18:70-79,1975.

[28] Neuberger JM, Davis M, Williams R. Clinical aspects of oral contraceptive associated liver tumours. In: Davis M, Tredger JM,Williams R,editors. Drug Reactions and the Liver.London: Pitman Medical. Pp 271-283,1981.

[29] Neuberger JM, Davis M.Advances in understanding of halothane hepatitis.TrendsPharmSci.;4:19-20,1983.

[30] Nickerson M, Ruedy J. Antihypertensive agents and the drug therapy of hypertension. In: Goodman LS, Gilman AG, Koelle GB, editors. The pharmacological basis of therapeutics.5th ed. Macmillan:New York. pp 1350$1368,1975$.

[31] Ostapowicz G, Fontana RJ, Schiødt FV. Results of a prospective study of acute liver failure at 17 tertiary care centers in the United States. Ann Intern Med. 137(12):947-954,2002.

[32] Prescott LF. Hepatotoxicity of mild analgesics. Br J Clin Pharmac.; 10:373-379,1980.

[33] Rao RR. Mechanism of drug induced hepatotoxicity. Ind J Pharmac.; 5(2):313-318,1973.

[34] Schaller JG. Chronic salicylate administration in juvenile rheumatoid arthritis: aspirin "hepatitis" and its clinical significance. Pediatr.; 62:916-925,1978.

[35] Sharma SK,Balamurugan A,Saha PK,Pandey RM, Mehra NK.Evaluation of clinical and immunogenetic risk factors for the development of hepatotoxicity during antituberculosis treatment. Am J Respir Crit Care Med.; 166:916-919,2002.

[36] Stolk MF, Becx MC, Kuypers KC, Seldenrijk CA. Severe hepatic side effects of ezetimibe. Clin Gastroenterol Hepatol.; 4(7):908-911,2006,

[37] Timbrell JA. Drug hepatotoxicity. Br J Clin Pharmac.; 15:3-14,1983.

[38] Tredger JM, Davis M. Drug metabolism and hepatotoxicity Gut.; 34-39,1991.

[39]Zimmerman HJ. Hepatotoxicity, New York: Appleton Century Crofts. 1978.

[40]Zimmerman H. Hepatic injury caused by therapeutic agents. In: Becker FF, editor. The Liver. New York: Marcel Dekker. pp 225-302,1974. 